\title{
RNA editing complex interactions with a site for full-round $U$ deletion in Trypanosoma brucei
}

\author{
ANASTASIA SACHARIDOU, CATHERINE CIFUENTES-ROJAS, KARI HALBIG, ALFREDO HERNANDEZ, \\ LAWRENCE J. DANGOTT, MONICA DE NOVA-OCAMPO, and JORGE CRUZ-REYES \\ Department of Biochemistry and Biophysics, Texas A\&M University, College Station, Texas 77843, USA
}

\begin{abstract}
Trypanosome $U$ insertion and $U$ deletion RNA editing of mitochondrial pre-mRNAs is catalyzed by multisubunit editing complexes as directed by partially complementary guide RNAs. The basic enzymatic activities and protein composition of these high-molecular mass complexes have been under intense study, but their specific protein interactions with functional pre-mRNA/gRNA substrates remains unknown. We show that editing complexes purified through extensive ion-exchange chromatography and immunoprecipitation make specific cross-linking interactions with A6 pre-mRNA containing a single ${ }^{32} \mathbf{P}$ and photoreactive 4-thioU at the scissile bond of a functional site for full-round $U$ deletion. At least four direct protein-RNA contacts are detected at this site by cross-linking. All four interactions are stimulated by unpaired residues just $5^{\prime}$ of the premRNA/gRNA anchor duplex, but strongly inhibited by pairing of the editing site region. Furthermore, competition analysis with homologous and heterologous transcripts suggests preferential contacts of the editing complex with the mRNA/gRNA duplex substrate. This apparent structural selectivity suggests that the RNA-protein interactions we observe may be involved in recognition of editing sites and/or catalysis in assembled complexes.
\end{abstract}

Keywords: Trypanosoma brucei; RNA editing; RNA-protein interactions; editing complexes

\section{INTRODUCTION}

Mitochondrial mRNAs in trypanosomatid protozoa including Trypanosoma, Leishmania, and Crithidia species undergo a unique form of RNA editing by cycles of uridylate insertion or deletion at numerous editing sites (ESs). This post-transcriptional mRNA maturation progresses with a general $3^{\prime}-5^{\prime}$ polarity and is catalyzed by a large multisubunit RNA editing complex (also termed $20 \mathrm{~S}$ editosome or L-complex) proposed to contain between 8 and 20 polypeptides depending on the purification protocol (Rusche et al. 1997; Panigrahi et al. 2001a, 2003; Aphasizhev et al. 2003a; Law et al. 2005). The smaller number presumably reflects high-stringency purification conditions and tight association of the subunits in the resulting complexes. Partially complementary guide RNA (gRNA) transcripts direct this process, which is believed to initiate with the formation of an "anchor duplex" with premRNA. Catalysis of a single editing cycle involves three

Reprint requests to: Jorge Cruz-Reyes, Department of Biochemistry and Biophysics, Texas A\&M University, 2128 TAMU, College Station, TX 77843, USA; e-mail: cruzrey@tamu.edu; fax: (979) 862-4718.

Article published online ahead of print. Article and publication date are at http://www.rnajournal.org/cgi/doi/10.1261/rna.2295706. basic activities, namely, mRNA endonuclease, 3' terminal uridylyl transferase (TUTase in insertion) or $3^{\prime}$ to $5^{\prime}$ U-specific exoribonuclease (in deletion), and RNA ligase. So far, the known catalytic subunits in the editing complex include a TUTase (KRET2, also termed LC-6b; Aphasizhev et al. 2003a; Ernst et al. 2003), a U-specific exonuclease (KREP6, LC-2; Kang et al. 2005), two RNA ligases (KREL1, band IV, LC-7a and KREL2, band V, LC-9; McManus et al. 2001; Rusche et al. 2001; Schnaufer et al. 2001), deletion and insertion endonucleases (KREN1 and KREN2; Trotter et al. 2005 and Carnes et al. 2005, respectively), and an endonuclease/exonuclease (KREPA3, band VI, LC-7b; Brecht et al. 2005). All these protein subunits have been cloned and characterized in vitro and in vivo.

A significant amount of information has been obtained on the structural and functional composition of editing complexes (for reviews, see Madison-Antenucci et al. 2002; Simpson et al. 2004; Stuart et al. 2005); however, the specific RNA-protein interactions in assembled complexes during recognition of pre-mRNA/gRNA duplex substrates and catalysis of full editing cycles are unknown. Several reported protein subunits contain conserved motifs for nucleic acid binding, but only a purified recombinant KREPA3 has been shown to exhibit RNA-binding activity 
(Brecht et al. 2005). In addition to core essential subunits, a few auxiliary components involved in editing are known, including the annealing factors MRP1 (gBP21) and MRP2 (gBP25) (Blom et al. 2001; Muller et al. 2001; Aphasizhev et al. 2003b; Vondruskova et al. 2005), and the gRNA-binding factor RBP16 (Pelletier and Read, 2003). Other proposed factors are an RNA helicase, REAP1, and TbRGG1 (Missel et al. 1997; Madison-Antenucci et al. 1998; Vanhamme et al. 1998; Panigrahi et al. 2003). All factors mentioned above are either weakly or not associated with editing complexes and dispensable for in vitro editing (Rusche et al. 1997; Allen et al. 1998; Aphasizhev et al. 2003a; Panigrahi et al. 2003). Here, using photocross-linking we report four protein interactions in intimate contact with the first editing site (ES1) for full-round $U$ deletion in an A6 pre-mRNA/gRNA substrate that copurify and coimmunoprecipitate with editing complexes. All four RNA-protein cross-links exhibit structural selectivity for the single-stranded character of the editing site region. Together, the data indicate that the cross-linking events described here are mediated by one or more stably bound core subunits. To our knowledge, this is the first report of specific RNA-protein interactions of editing complexes with a functional site for full-round RNA editing.

\section{RESULTS}

To search for RNA-protein interactions in assembled RNA editing complexes, we generated a 72-nt A6 pre-mRNA substrate containing a single ${ }^{32} \mathrm{P}$ and 4-thioU at the scissile bond of the first editing site (ES1) for $U$ deletion (Fig. 1A). Prior to photocross-linking, this thiolated pre-mRNA was preannealed with gRNA and mixed with editing complex preparations, as in standard in vitro reactions (see Materials and Methods). Importantly, the thiolated pre-mRNA supports accurate in vitro deletion of three uridylates as directed by the partially complementary gRNA D33 (Cruz-Reyes et al. 2001), although slightly less efficiently than unmodified pre-mRNA (Fig. 1A,B). This indicates that the presence of a thio-uridylate immediately $3^{\prime}$ of the scissile bond does not significantly interfere with editing activity.

We initially utilized crude mitochondrial lysate that was fractionated by
Q-sepharose chromatography (Q1 column; Fig. 1C) to detect protein cross-links to ES1. This column has been previously used to enrich active editing complexes (Rusche et al. 1997; Panigrahi et al. 2001a; Pai et al. 2003). Several cross-links of various intensities are evident across the fractionated lysate upon irradiation with 365-nm UV light. At least four of them, at about 40, 50, 60, and $100 \mathrm{kDa}$, appeared to closely copurify with editing complexes as detected by immunoblots of known core subunits, particularly in the peak fractions 9-11 ("peak Q1 fractions"; Fig. 1C,D). Other prominent cross-links were detected at about 75,150 , and $250 \mathrm{kDa}$ in or near these fractions. Proteinase $\mathrm{K}$ inactivation of all cross-links in the peak Q1 fractions showed that they are protein dependent (not shown), so from here onward we will refer to them as

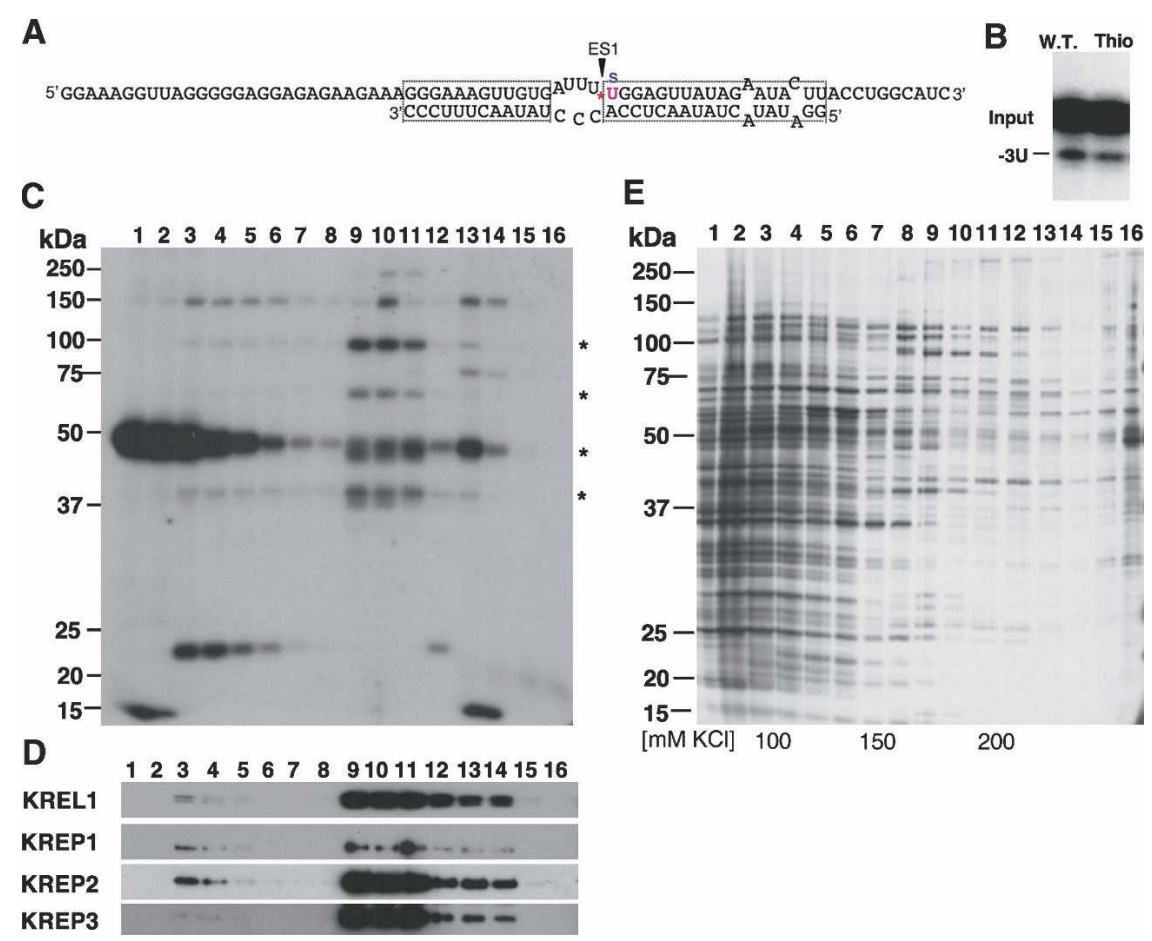

FIGURE 1. RNA-protein interactions detected by photocross-linking with a pre-mRNA/ gRNA substrate for full-round deletion copurify with editing complexes in Q-sepharose fractionated mitochondrial extract. (A) Diagram of the 72-nt A6 pre-mRNA substrate annealed with 33-nt gRNA D33 used in this study. The boxes indicate the predicted upstream and downstream duplexes flanking the first editing site (ES1) for full-round deletion. The 4-thioU $\left({ }^{s} \mathrm{U}\right)$ and $5^{\prime}{ }^{32} \mathrm{P}$-radiolabed bond $\left({ }^{*}\right)$ are positioned at the double-strand/single-strand junction that defines ES1 (indicated by an arrowhead). (B) Full-round U deletion in vitro assay of the unmodified wild-type (W.T.) and thiolated A6 pre-mRNA paired with gRNA D33. The input and accurate $3 \mathrm{U}$ deletion RNAs are indicated. $(C)$ Long UV irradiation $(365 \mathrm{~nm})$ of the pre-mRNA/gRNA substrate with all Q-sepharose fractions. The asterisks indicate the positions of four proteinase K-sensitive cross-links that consistently copurify with editing complexes. Editing complexes were detected in immunoblots $(D)$ of four known subunits KREL1 (also termed TbMP52, band IV, LC-7c), KREP1 (TbMP81, band II, LC1), KREPA2 (TbMP63, band III, LC4), and KREPA3 (TbMP42, band VI, LC-7b). Editing complexes and copurifying crosslinks peak in fractions 9-11. The molecular size of protein markers is indicated in kilodaltons. For current listings and nomenclature of the known subunits in different labs see Stuart et al. (2005) and Simpson et al. (2004). (E) Silver-staining of all Q1 sepharose fractions in C. The peak Q1 fractions elute between 150 and $200 \mathrm{mM} \mathrm{KCl}$. 
p40, p50, p60, and p100. The peak Q1 fractions eluted away, between 150 and $200 \mathrm{mM} \mathrm{KCl}$, from most proteins in the mitochondrial crude extract, and therefore appear significantly enriched (Fig. 1E). These fractions were pooled and further purified by two subsequent steps of ion-exchange chromatography in DNA-cellulose and Qsepharose columns, respectively (Fig. 2; data not shown). Notably, p40, p50, p60, and p100 copurify with editing activity in both columns. However, additional bands are detected in the DNA-cellulose column ("D") peak fractions, although not reproducibly in our protein preparations (not shown). The peak fractions of the second $Q$ sepharose column ("Q2" fractions 13-15) show primarily p40, p50, p60, and p100 (Fig. 2A), precisely copurifying with isolated silver-stained polypeptides and full-round deletion activity (Fig. 2B,C). Notably, our peak Q2 fractions exhibit a pattern of major stained protein bands, plus a few additional fainter bands (Fig. 2B), that is remarkably similar to that of editing complexes purified with either the same protocol (Sollner-Webb et al. 2001) or another biochemical purification strategy (Panigrahi et al. 2001a).

A

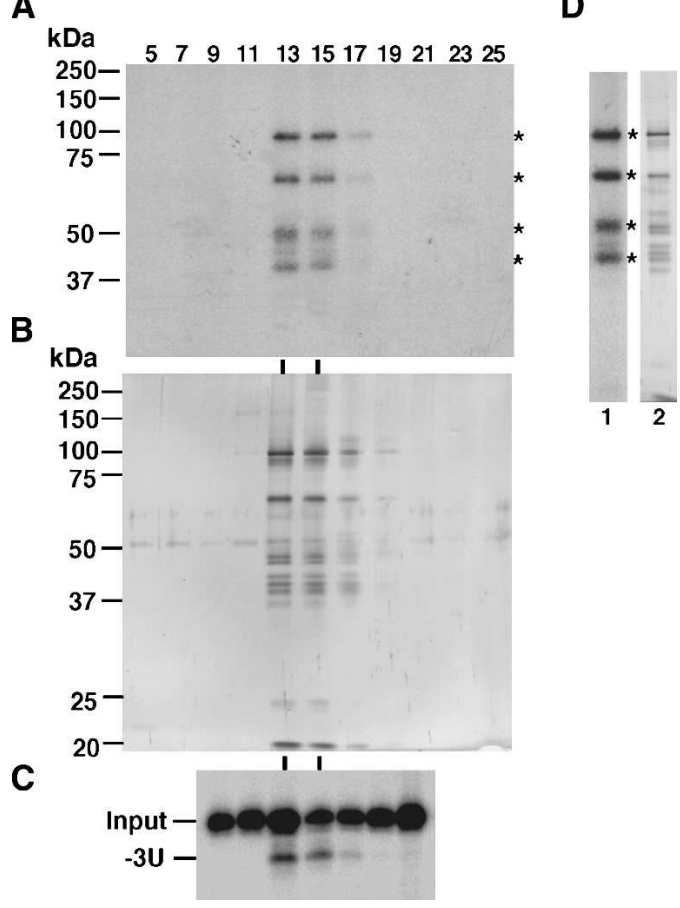

FIGURE 2. p40, p50, p60, and p100 copurify with editing complexes after extensive ion-exchange chromatography. The peak Q1 fractions from Figure 1 were subsequently fractionated on DNA cellulose (D) and a second Q sepharose column (Q2). Shown are the relevant odd fractions of the Q2 elution. The four protein-RNA cross-links $(A)$ precisely copurify with silver-stained editing complexes $(B)$ and their $\mathrm{U}$ deletion activity $(C)$. The $\mathrm{U}$ deletion activity of the $\mathrm{Q} 2$ fractions was assayed at the ES1 of the $3^{\prime}$-end-labeled A6 pre-mRNA. The RNA input and accurate deletion product $(-3 \mathrm{U})$ are indicated. $(D)$ The four protein-RNA cross-links (lane 1) colocalize with silver-stained protein components (lane 2) of the peak Q2 fractions (no. 13-15).

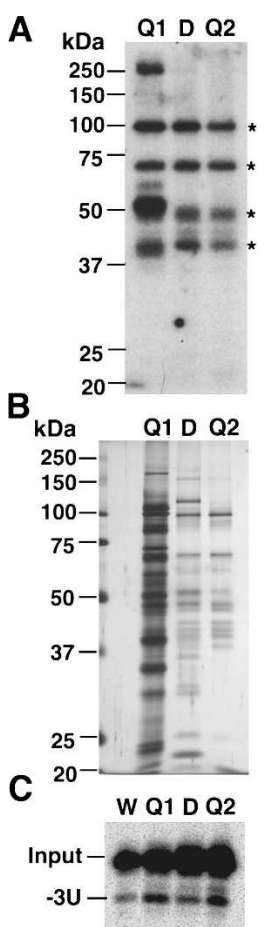

FIGURE 3. Side-by-side gel analyses of Q1, D, and Q2 peak fractions. (A) protein-RNA cross-linking interactions, $(B)$ silver staining, and (C) full-round U-deletion activity. The latter includes a lane with the original whole mitochondrial extract $(\mathrm{W})$.

Both the same protein pattern and relative intensity of individual bands are conserved whether silver or sypro ruby staining is used (data not shown). Importantly, p40, p50, p60, and p100 colocalize with stained bands in the Q2 peak fractions (Fig. 2D). Furthermore, these cross-links are only detected if the targeted residue is thiolated and, therefore, upon $365-\mathrm{nm}$ but not $260-\mathrm{nm}$ UV light irradiation (data not shown).

A direct comparison of the protein content and crosslinking pattern of Q1, D, and Q2 peak fractions (Fig. 3A,B) indicates that the four RNA-protein interactions described above are conserved throughout the purification of active editing complexes (Fig. 3C) and most likely involve the same proteins. Other cross-links previously observed in Q1 and occasionally in $\mathrm{D}$ fractions are significantly reduced or lost in Q2 fractions. The peak Q2 fractions (13-15) contain $\sim 1 / 6000$ of the original crude mitochondrial extract protein and exhibit a simpler protein pattern than the parental $\mathrm{D}$ and Q1 fractions. This extent of purification is consistent with others reported using similar protocols (Rusche et al. 1997; Panigrahi et al. 2001a; Oppegard and Connell 2002). There is at least an $\sim 10$-fold further purification compared to the whole-cell protein content; however, the specific activity of editing complexes could not be calculated since the in vitro editing assay is not linear with protein added, particularly in cruder fractions (Rusche et al. 1997; Panigrahi et al. 2001a; Oppegard and Connell 2002; data 
not shown). Together, these data suggest that p40, p50, p60, and p100 are tightly associated with purified active editing complexes and that they make intimate contacts with the targeted editing site.

To further confirm this association, we performed coimmunoprecipitation assays (co-IP) using monoclonal antibodies that are known to immunoprecipitate active editing complexes (Panigrahi et al. 2001a,b). Analysis of the peak Q1 fraction shows efficient co-IP of the p40, p50, p60, and p100 kDa cross-links by anti-KREPA3 antibodies (Fig. 4). Relative to a control lane showing the starting cross-linked sample ("C"), the unbound lane ("U") shows a significant decrease in three cross-links, p40, p60, and p100, and their corresponding enrichment in the bound material (" $\mathrm{B}$ ") after two washes ("W2"). Most cross-linking activity at $\sim 50 \mathrm{kDa}$ remains in the unbound fraction, but a significant amount (above background levels) co-IPs with the editing complex, as compared with a mock assay with no antibodies. We interpret this as indicative of at least two proteins comigrating at $\sim 50 \mathrm{kDa}$, one corresponding to a stably bound component (p50) of editing complexes and another representing a mitochondrial protein that is presumably abundant but not tightly associated with editing complexes. Consistent with this notion, the latter cross-link may account for the prominent $\sim 50-\mathrm{kDa}$ band in the flowthrough and first few fractions in the initial chromatographic step (Fig. 1C), and apparent trailing into the peak editing fractions. The same cross-linking protein is significantly reduced or lost in the D and Q2 peak fractions (Fig. $3 \mathrm{~A}$ ), and in most gels, it appears to migrate slightly above the proposed p50 cross-link (e.g., Figs. 1C, 3A, 4). Co-IP assays were also performed with antibodies against two other editing subunits, KREPA2 and KREL1, and in both cases p40, p50, p60, and p100 selectively immunoprecipitate with editing complexes (not shown).

Additional analyses were performed to confirm the specificity of the p40-100 interaction with editing complexes. These include a positive control showing efficient co-IP of radiolabeled RNA ligase subunits (via ${ }^{32} \mathrm{P}$-adenylylation; Panigrahi

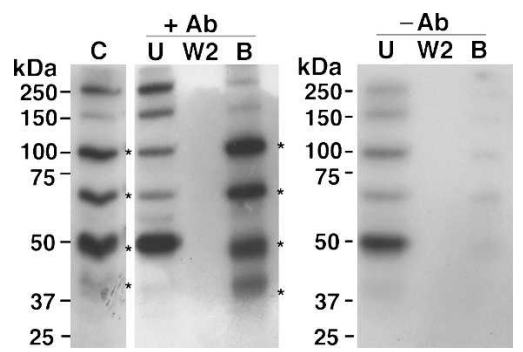

FIGURE 4. p40, p50, p60, and p100 coimmunoprecipitate with editing complexes. Protein-RNA cross-links in a peak Q1 fraction before (C lane) and after a co-IP assay with anti-KREPA3 antibodies $(+\mathrm{Ab})$, including the unbound $(\mathrm{U})$, second wash (W2), and bound immunoprecipitated (B) fractions. A parallel mock co-IP assay with no antibodies $(-\mathrm{Ab})$ is shown. et al. 2001a,b) and a negative control with a nonrelated antibody (not shown). The virtual absence of the $\sim 150$ - and $\sim 250$-kDa cross-links in Q2 fractions (Fig. 2A) and their reduction to near background levels in co-IP assays (Fig. 4) suggest that the cross-linking proteins are either weakly or not bound to editing complexes.

Combined, our extensive chromatography purification and immunoprecipitation analyses show at least four RNAprotein cross-links between one or more stably bound subunits of editing complexes and a site for full-round deletion in an A6 substrate. Notably, these cross-links specifically target the $\left[{ }^{32} \mathrm{P}\right]$-labeled photoreactive 4 -thioU positioned at the scissile bond of this functional substrate.

To determine whether or not the polypeptides that bind ES1 also contact other positions of the A6 pre-mRNA/ gRNA substrate, we moved the $\left[{ }^{32} \mathrm{P}\right]$-labeled photoreactive 4-thioU a few nucleotides away from the scissile bond at ES1 (bond 45; Fig. 5A). In one case, we tested the upstream bond 34 that corresponds to the second deletion site (ES4) in the natural A6 substrate, and in another, the downstream bond 51 in the never-edited region of this transcript. Both positions are located within the predicted upstream and downstream duplexes formed by the partially complementary gRNA D33, respectively (Fig. 5A, top and middle RNA pairs). Notably, all four protein-RNA interactions detected by cross-linking at functional ES1 (bond 45) are absent at either duplex position (Fig. 5B). This suggests that the observed RNA-protein cross-linking interactions may exhibit structural selectivity for single-strandedness of the editing site. To confirm this apparent preference for singlestranded residues adjoining the photoreactive 4-thioU, we annealed the pre-mRNA to a gRNA derivative (31.dx) that extends the upstream and downstream duplexes into a single contiguous duplex (Fig. 3A, bottom pair). We found that base-pairing of the ES1 region with 31.dx strongly inhibits all cross-links observed with the parental gRNA D33 (Fig. 5C).

Together, our data indicate that all four cross-linking proteins observed at ES1 are favored by the single-strand character of the editing site. Importantly, precise gRNA base-pairing across ES1 inhibits in vitro U deletion at this site (Cruz-Reyes and Sollner-Webb, 1996).

To assess the specificity of the interaction between editing complexes and A6 pre-mRNA/D33 substrate, we supplemented the cross-linking assay with a molar excess of various nonradiolabeled RNA competitors (Fig. 6A-C). Interestingly, addition of 10- and 25-fold excess (relative to radiolabeled A6 pre-mRNA) of the homologous A6 premRNA virtually abolished all cross-linking (Fig. 6A, lanes 1-3), whereas another pre-mRNA (CYb; lanes 4-5) and tRNA (lanes 6-7) were only slightly inhibitory at the same concentration. The partial effect of the latter heterologous competitors seems specific to these transcripts, as further addition (25-fold) of gRNA D33 did not affect the crosslinking efficiency (lanes 8-9). Note that the assay includes 


\section{A}

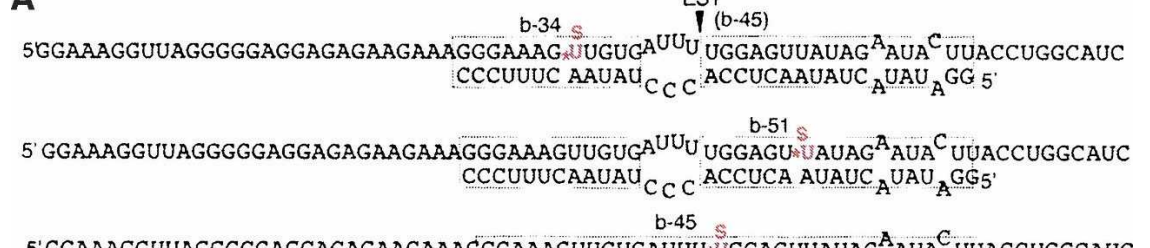
5' GGAAAGGUAGGGGGAGGAGAGAAGAAAGGGAAAGUGUGAUUU «GGAGUAUAG ${ }^{\circledR}$ AUA ${ }^{C}$ UUACCUGGCAUC CCUUUCAAUAUUAGA GCCUCAAUAUC $\mathrm{A}_{\mathrm{UAU}} \mathrm{GG}_{5}$

B

$32 \mathrm{P}$ bond 344551

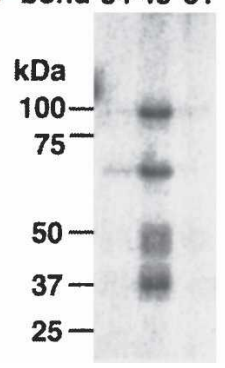

C

gRNA D33 31.dx

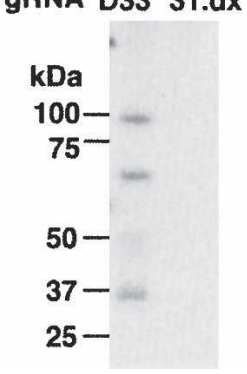

FIGURE 5. All four RNA-protein interactions detected by cross-linking in Pf editing complexes are favored by single-strandedness at the editing site. (A) Diagrams of A6 premRNA/D33 pairs as in Figure $1 \mathrm{~A}$, but with the $\left[5^{\prime}{ }^{32} \mathrm{P}\right]$ thiolated $\mathrm{U}$ at upstream $(\mathrm{b}-34)$ or downstream (b-51) bonds (top and middle RNA pairs, respectively). The position of ES1 (b-45) is also indicated. The A6 pre-mRNA modified at b- 45 was also paired to a gRNA D33 derivative (31- $\mathrm{dx}$ ) that forms a continuous duplex across ES1 (bottom pair). (B) Parallel crosslinking assays in a Q2 peak fraction of radiolabeled pre-mRNA at each of three indicated bonds above, paired with gRNA D33. (C) Cross-links of pre-mRNA modified at b-45 and annealed with either D33 or a D33-like derivative (31-dx) that fully base-pairs the ES1 and directs no deletion.

gRNA D33 at $\sim 100$-fold excess relative to the labeled premRNA (Cruz-Reyes et al. 2001; see Materials and Methods section). The inhibition by the A6 pre-mRNA competitor is consistent with its ability to base-pair with gRNA D33. Additional heterologous transcripts including the noncomplementary gRNA gRPS12, viral RNA H121 (25- to 50-fold excess), and several homopolymers (100-fold excess) were slightly or not inhibitory (Fig. 6B,C; data not shown). Up to 100 -fold further addition of gRNA D33 (i.e., $\sim 200$-fold excess overall) in the latter assays was not inhibitory (Fig. 6B, lanes 5,6).

We also tested the above RNA competitors on full-round $\mathrm{U}$ deletion. As expected, the homologous pre-mRNA was fully inhibitory at 25-fold excess, whereas all other competitors in Figure 6A-C were little or not inhibitory at the same concentration (Fig. 6D; data not shown). Combined, the above competition analyses on cross-linking and editing assays suggest that editing complexes may be able to distinguish the pre-mRNA/gRNA duplex from individual substrate strands and from nonrelated structured or relatively nonstructured transcripts. Additional studies are currently under way in our laboratory to further address this question.

Based on the observed gel mobility of p40, p50, p60, and p100, we suspected that one or more of them could correspond to known subunits of editing complexes. To test this possibility, we transferred the reactions to a membrane after cross-linking and performed Western analysis using available monoclonal antibodies to identify the colocalizing proteins. Our initial analysis showed a precise colocalization between p60 and KREPA2 ( 60 kDa; band III; LC-4), whereas p40 did not precisely match with KREPA3 ( $\sim 40 \mathrm{kDa}$; band VI; LC-7b) (Fig. 7). Furthermore, p40 and p50 do not comigrate with the editing RNA ligases $\left({ }^{32} \mathrm{P}\right.$-labeled by adenylylation; Sabatini and Hajduk 1995; data not shown). MS analyses of the protein bands matching the crosslinks are under way, but due to the possibility of cross-contamination between similar-size subunits (particularly in the $\sim 90-100 \mathrm{kDa}$ and $\sim 40-55 \mathrm{kDa}$ size ranges; Stuart et al. 2005) additional work using epitope-tagging of candidate subunits will be required to establish definite subunit assignments for $\mathrm{p} 40$, p50, and p100, and confirm that p60 corresponds to KREPA2.

Overall, the extensive biochemical copurification and coimmunoprecipitation of p40, p50, p60, and p100 with active editing complexes indicates that the cross-links involve one or more stably bound components of editing complexes. Moreover, our analysis of substrate features and response to RNA competitors suggests that editing complexes and possibly these particular RNA-protein interactions exhibit structural selectivity for the editing substrate used in our studies.

\section{DISCUSSION}

The specific RNA-protein interactions in editing complexes that lead to their activation and catalysis of faithful RNA editing cycles in trypanosomes are unknown. The purpose of this study was to identify specific pre-mRNA/ protein contacts using assembled editing complexes and an A6 pre-mRNA/gRNA substrate for full-round editing in vitro. We found at least four protein interactions, p40, p50, p60, and p100, in direct contact with ES1 for U deletion. These interactions revealed by protein-RNA cross-linking involve one or more tightly bound subunits of editing complexes since they precisely copurify with editing activity upon extensive ion-exchange chromatography in three consecutive columns and co-IP using monoclonal antibodies raised against known editing complex subunits. The ion-exchange chromatography (Sollner-Webb et al. 2001) 
A

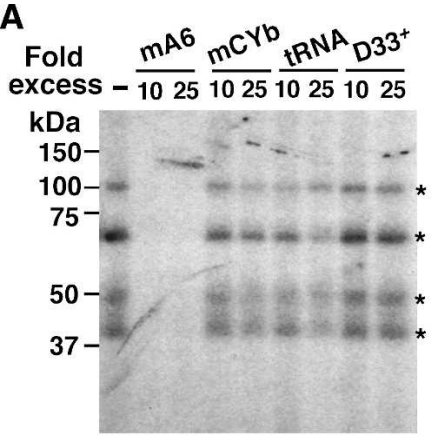

D

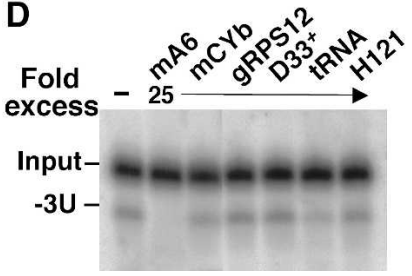

B

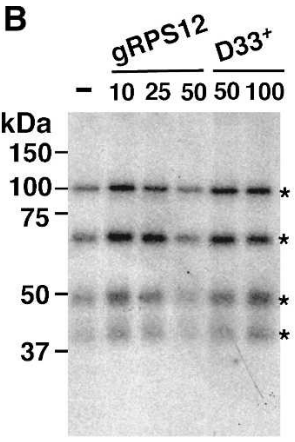

C

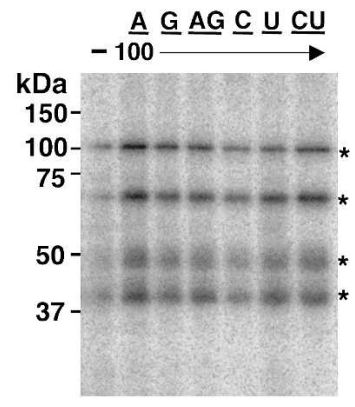

enzymatic steps of $U$ deletion. Furthermore, this subunit has also been proposed to play a critical structural role in the formation or stability of entire editing complexes (Huang et al. 2002; Kang et al. 2004). Other reported subunits of predicted molecular size similar to p60, although not found during the peptide sequencing of band III (by Edman degradation; Huang et al. 2002), include KREN2 and KREPB2, an essential insertion-specific endonuclease and a potential endonuclease, respectively (Carnes et al. 2005; Trotter et al. 2005). At least the essential KREN2 is expected in our purified complexes, either migrating with band III (possibly at substoichiometric levels) or near to it. Another reported subunit, KRET2, appeared to be substoichiometric (Law et al. 2005) in similarly purified complexes.

p100 precisely colocalizes with the prominent band I (Rusche et al. 1997), which corresponds to an $(\sim 99 \mathrm{kDa})$ exonuclease proposed to function in $\mathrm{U}$ deletion (KREPC2; LC-3; Simpson et al. 2004; Stuart et al. 2005). However, we cannot exclude the possibility that

and immunoprecipitation (Panigrahi et al. 2001a,b) approaches applied in this study were previously exploited to efficiently purify active editing complexes and study their protein composition. All major protein components of the complexes originally observed by Rusche et al. (1997) are also present in the complexes prepared by immunoprecipitation and similar chromatography or affinity purifications (Aphasizhev et al. 2003a; Panigrahi et al. 2001a,b, 2003).

The identification of the cross-linking polypeptides reported is evidently necessary to begin dissecting their potential editing. The protein banding pattern of our purified editing complexes is remarkably similar to others previously reported using related biochemical purification schemes (Rusche et al. 1997; Panigrahi et al. 2001a, 2003), and associations between specific subunits and protein bands in those patterns have been proposed (for reviews, see Simpson et al. 2004; Stuart et al. 2005). Based on the colocalization of p60 with band III (KREPA2; LC-4) in both silver-stained gels (Fig. 2D) and immunoblots (Fig. 7; data not shown) we speculate that p60 may indeed correspond to band III. The precise molecular function of this subunit has not been defined, but it has been found associated with KREPC2 and KREL1 in a purified subcomplex that catalyzes partial (precleaved) deletion editing (Schnaufer et al. 2003). These authors have speculated that KREPA2 could use its potentially regulatory $\mathrm{OB}$ fold to coordinate the sequential p100 may be the closely migrating KREN1, an essential U deletion-specific endonuclease (Panigrahi et al. 2003) expected in our purified active complexes, or alternatively KREPC1 $(\sim 100 \mathrm{kDa})$, a candidate editing exonuclease (Panigrahi et al. 2003) potentially present in our preparation. Any of the above likely p100 candidates is consistent with our search for subunits that bind and cross-link a deletion site.

Several known editing complex subunits could account for the p40 and p50 cross-links we observe (Simpson et al. 2004; Stuart et al. 2005), including five ( $\sim 41-$ to $49-\mathrm{kDa})$ subunits with a conserved U1-like Zn-finger domain potentially involved in macromolecular interactions with RNA

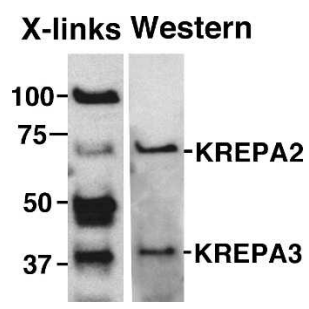

FIGURE 7. p60 colocalizes with the KREPA2 subunit. A cross-linking reaction (X-links lane) and subsequent Western blot analysis of the same gel (Western lane) with anti-KREPA2 and anti-KREPA3 antibodies. 
substrates or other proteins in the complex. Two of these proteins also exhibit a C-terminal Pumilio RNA-binding domain and less conserved RNase III motifs potentially involved in endonuclease cleavage. Our Western blot analysis revealed that p40 is not KREPA3 $(\sim 42 \mathrm{kDa}$; Fig. 7). Moreover, the RNA ligases KREL1 $(\sim 52 \mathrm{kDa})$ and KREL2 $(\sim 45 \mathrm{kDa})$ migrate between the p40 and p50 cross-links in high-resolution acrylamide gels and therefore are different proteins (not shown). It is also conceivable that one or more of these proteins, $\mathrm{p} 40, \mathrm{p} 50$, and/or p100, correspond to novel subunits of editing complexes. Further work is under way to identify these proteins and their potential roles in deletion.

KREPA3 ( $\sim 42-\mathrm{kDa}$ subunit) and five related subunits exhibit apparent $\mathrm{Zn}$-finger domains and/or an $\mathrm{OB}$ fold. The former are found in many regulatory proteins and could mediate interactions with nucleic acids or with other proteins, whereas the latter typically provides a nonspecific binding platform for single- and double-stranded nucleic acids (Suck 1997). KREPA3 is the only subunit known so far to bind RNA (Brecht et al. 2005). Surprisingly, a recombinant version of this protein was reported to exhibit endonuclease and $3^{\prime}-5^{\prime}$ exonuclease activities on a stretch of unpaired uridylates in a partial RNA hybrid, although KREPA3 lacks recognizable nuclease domains. While these activities are editing-like, the substrate used in that study is not functional, and the proposed proteinRNA interaction remains to be confirmed in assembled editing complexes. RNAi knockdown of KREPA3 does not appreciably disassemble editing complexes, but reduces in vivo and in vitro editing (Brecht et al. 2005). Thus, the reported properties of rKREPA3 suggest that this subunit has important roles in editing. Whether or not KREPA3 is functionally similar or even redundant to any structurally related subunit remains to be determined. KREPA3 was not detected in our analysis at ES1, however this may reflect a limitation of our "zero-distance" cross-linking approach. That is, even if a protein specifically binds the targeted site, the thiolated uridylate and adjacent amino acid side chain may not be properly orientated with each other for efficient photoreaction.

A double-strand/single-strand junction just $5^{\prime}$ of the of the dowstream "anchor" duplex is a critical feature of functional editing sites (Seiwert et al. 1996; Cruz-Reyes and Sollner-Webb 1996). Interestingly, the cross-links we observe are strongly inhibited by gRNA base-pairing of the editing site (Fig. 5). This observation suggests that the p40100 interactions with the substrate exhibit structural selectivity for the mismatched preedited ES1, but are inhibited by gRNA complementarity across the edited site. In addition to simple mRNA/gRNA mismatches at editing sites, structural studies have indicated that other features of functional pre-mRNA/gRNA pairs may determine the basis for endonuclease recognition (Leung and Koslowsky 2001). Nevertheless, it is feasible that p40, p60, p50, and p100 may play important roles during recognition and/or catalysis at editing sites. A previous study of $\mathrm{U}$ insertion in Leishmania proposed that two RNA cross-linking proteins, $\sim 80$ and $100 \mathrm{kDa}$, from highly enriched editing extracts may be associated with editing site recognition, but the RNA substrate positions cross-linked remain to be determined (Oppegard et al. 2003).

Our competition analyses also suggest that editing complexes may preferentially recognize features of the pre-mRNA/gRNA hybrid (Fig. 6). gRNA D33 is supplemented at $\sim 100$-fold the level of the radiolabeled A6 premRNA, in both standard cross-linking and editing assays, although we have seen that a $\sim 200$-fold excess affects neither activity (Fig. 6B,D). Importantly, we have seen in native gels that during the preincubation step in our assays virtually all radiolabeled A6 pre-mRNA anneals to gRNA D33 (see Materials and Methods section; data not shown). Addition of nonradiolabeled A6 pre-mRNA at 10-fold excess (or less) strongly inhibits cross-linking and editing (Fig. 6A; data not shown), whereas 25- to 100 -fold excess of other transcripts that should not hybridize with gRNA D33 have little or no effect. Interestingly, significantly structured transcripts such as tRNA (25-fold) appear relatively more inhibitory than predicted low-structured sequences, including the gRNA constructs (50-fold) and short RNA homopolymers (100-fold) tested (Fig. 6; data not shown). This apparent binding preference of editing complexes for RNA substrates in vitro is under further investigation in our laboratory.

Our observation of multiple cross-linking interactions at the ES1 for deletion in the A6 pre-mRNA/gRNA substrate may reflect that this site is dense with protein contacts in editing complexes (possibly not all detected by our cross-linking approach). Also the natural dynamics of interacting subunits, variable RNA substrate conformations, or protein breakdown may account for the multiple cross-links detected. These possibilities will be further studied in our laboratory. Furthermore, we observed the same cross-linking pattern in immunoprecipitated editing complexes enriched from bloodstream form trypanosomes (Halbig et al. 2004; data not shown). Together with our extensive purification of the procyclic complexes, this suggests that these proteins are part of the core complex and may not directly account for developmental regulation.

Finally, editing complexes contain subgroups of apparently related subunits sharing similar conserved motifs (Stuart et al. 2005). This may reflect the proposed functional and structural partition of insertion and deletion components in editing complexes (Cruz-Reyes et al. 1998a,b, 2002; Huang et al. 2001; Schanufer et al. 2003), and functions outside editing, including polycistronic mRNA, gRNA, and rRNA processing (Koslowsky and Yahampath 1997; Grams et al. 2000). Whether the editing complex cross-links reported here and/or other subunits occur at 
different deletion or insertion sites and in other substrates is currently under investigation in our laboratory.

\section{MATERIALS AND METHODS}

\section{Pre-mRNA and gRNA substrates}

The ATPase 6 (A6) pre-mRNA editing substrates (Seiwert et al. 1996) for deletion with gRNA D33 (Cruz-Reyes et al. 2001) were prepared as previously described. The site-specific radiolabeled and 4-thioU modified pre-mRNAs were obtained by ligation of two fragments as in Reichert et al. (2002). For bond 45 (ES1), the acceptor and donor RNAs were $5^{\prime}$-GGAAAGGUUAGGGGGAG GAGAGAAGAAAGGGAAAGUUGUGAUUU- ${ }^{\prime}$ and $5^{\prime}$-UGGA GUUAUAGAAUACUUACCUGGCAUC- $3^{\prime}$, the latter containing a $5^{\prime}$-terminal 4 -thioU in bold. For bond 34 (ES4), 5'-GGAAAG GUUAGGGGGAGGAGAGAAGAAAGGGAAAG and $5^{\prime}$-UUGU GAUUUUGGAGUUAUAGAAUACUUACCUGGCAUC- ${ }^{\prime}$; ; and bond 51, 5' -GGAAAGGUUAGGGGGAGGAGAGAAGAAAGGGA AAGUUGUGAUUUUGGAGU-3' and 5'-UGGAGUUAUAGAA UACUUACCUGGCAUC- $3^{\prime}$ were used, respectively.

The acceptor RNAs were transcribed using the Uhlenbeck single-stranded T7 transcription method (Milligan et al. 1987) and gel purified. The donor thiolated RNAs were chemically synthesized by Dharmacon. The 4-thioU residue of the donor piece was radiolabeled to high-specific activity with polynucleotide kinase and $\left[\gamma^{-}{ }^{32} \mathrm{P}\right] \mathrm{ATP}$ (using a 1:2 molar ratio of $5^{\prime}$ ends:ATP), gel purified, and ligated to the acceptor piece using the following DNA oligonucleotide bridges (bond 45): 5' -TATTC TATAACTCCAAAATCACAACTTTCC-3'; (bond 34), 5' -AACTC CAAAATCACAACTTTCCCTTTGTTC-3'; (bond 51), 5' -GCCAG GTAAGTATTCTATAACTCCAAAATC-3'. A 3:1:2 molar ratio of acceptor/donor/bridge molecules was used.

\section{Preparation of crude mitochondrial extracts and fractions containing enriched or purified editing complexes}

Procyclic form (Pf) T. brucei strain TREU667 was grown in Cunningham media, and mitochondrial crude extracts were prepared as in Harris and Hajduk (1992), with modifications as in Sollner-Webb et al. (2001). Mitochondrial crude extracts were fractionated by ion-exchange chromatography in consecutive Q-sepharose (Q1) DNA-cellulose (D), and Q-sepharose (Q2) columns, as described by Rusche et al. (1997) and Sollner-Webb et al. (2001). The elution fractions with the peak of editing complexes determined by Western blot analysis or editing activity also contained the peak of cross-linking activity in all purification steps.

\section{Editing, adenylylation, and cross-linking analysis}

Full-round editing reactions assembled in $20-\mu \mathrm{L}$ mixtures with preannealed 3 ' -end labeled A6 pre-mRNA $(\sim 10 \mathrm{fmol})$ and gRNA D33 $(\sim 1.2 \mathrm{pmol})$ and adenylylation of RNA ligases in editing complexes were performed as in Cruz-Reyes et al. (1998a,b) and Sabatini and Hajduk (1995), respectively. For photocross-linking analysis, editing reactions were assembled as above, but in the absence of nucleotides, which somewhat improves cross-linking. The complete mixtures were incubated for $10 \mathrm{~min}$ at $26^{\circ} \mathrm{C}$ and an additional $10 \mathrm{~min}$ on ice prior to irradiation with 365-nm UV light (on ice for $10 \mathrm{~min}, \sim 5 \mathrm{~cm}$ below a Spectroline $150-\mathrm{V}$ lamp) and subsequent treatment with RNases A and T1 $(50 \mu \mathrm{g} / \mathrm{mL}$ and $120 \mathrm{U} / \mathrm{mL}$ ) for $10 \mathrm{~min}$ at $37^{\circ} \mathrm{C}$. After addition of $7 \mu \mathrm{L}$ of $4 \times$ Laemmli buffer, the samples were analyzed by SDS-PAGE and autoradiography. RNA competitors at the indicated molar excess were included in the reaction mixture supplemented to the preannealed pre-mRNA/gRNA duplex in both cross-linking and editing assays. The 15-nt homopolymers were synthesized by IDT. The 121-nt viral RNA H121 was a gift from Cheng C. Kao (Hema and Kao 2004). We have determined in native gels that our preannealing step yields $>95 \%$ of the pre-mRNA in a duplex with gRNA D33 (not shown), so further gRNA addition in Figure 6A,B should hybridize virtually all pre-mRNA.

\section{Immunoprecipitation and Western blot analysis}

Immunoprecipitations were performed essentially as described by Panigrahi et al. (2001a) with minor modifications. For immunoprecipitation analysis of cross-linking proteins, editing reactions were scaled up 10 times and cross-linked as described above. One hundred microliters of Immunomagnetic beads (Dynabeads M-450; Dynal) were coupled with $225 \mu \mathrm{L}$ of monoclonal antibodies (kindly provided by the laboratory of Ken Stuart, SBRI Seattle) and $1 \%$ BSA. Editing reactions were incubated with antibodycoated beads for $1 \mathrm{~h}$ at $4^{\circ} \mathrm{C}$ using a biodirectional shaker and occasional tapping. After washing two times with $100 \mu \mathrm{L}$ of immunoprecipitation buffer $(10 \mathrm{mM}$ Tris at $\mathrm{pH} 7.2,10 \mathrm{mM}$ $\mathrm{MgCl}_{2}, 200 \mathrm{mM} \mathrm{KCl}, 0.1 \%$ Triton-X 100) the beads were resuspended with $100 \mu \mathrm{L}$ of TE buffer and incubated in the presence of RNases $\mathrm{A}$ and $\mathrm{T} 1$ as described above. Upon the addition of $30 \mu \mathrm{L}$ of $4 \times$ Laemmli buffer, the bead suspension was boiled at $100^{\circ} \mathrm{C}$ for $5 \mathrm{~min}$ and the supernatant analyzed by SDSPAGE and autoradiography. The entire $200 \mu \mathrm{L}$ unbound fraction and $100 \mu \mathrm{L}$ washes mixed with $60 \mu \mathrm{L}$ and $30 \mu \mathrm{L}$ of $4 \times$ Laemmli buffer, respectively, boiled as well as analyzed. For Western blot analysis with the indicated monoclonal antibodies, protein samples (cross-linked to RNA or not) were separated by SDS-PAGE, blotted, and probed with the indicated mouse monoclonal antibodies at a dilution of 1:25-1:50. The secondary antibody was applied at a 1/5000 dilution and the blot developed using the ECL plus system (Amersham).

\section{ACKNOWLEDGMENTS}

We thank Laurie K. Read and members of the Cruz-Reyes laboratory for comments on the manuscript and helpful discussions. The monoclonal antibodies against subunits of the editing complex were kindly provided by Ken Stuart and Aswini Panigrahi (SBRI, Seattle). Daniel Osterwisch provided expert technical assistance. The viral transcript $\mathrm{H} 121$ was a gift from Cheng C. Kao. This work was supported by a grant from the National Institutes of Health, Grant GM067130 (to J.C.-R.).

Received November 15, 2005; accepted April 3, 2006.

\section{REFERENCES}

Allen, T.E., Heidmann, S., Reed, R., Myler, P.J., Goringer, H.U., and Stuart, K.D. 1998. Association of guide RNA binding protein 
gBP21 with active RNA editing complexes in Trypanosoma brucei. Mol. Cell. Biol. 18: 6014-6022.

Aphasizhev, R., Aphasizheva, I., Nelson, R.E., Gao, G., Simpson, A.M., Kang, X., Falick, A.M., Sbicego, S., and Simpson, L. 2003a. Isolation of a U-insertion/deletion editing complex from Leishmania tarentolae mitochondria. EMBO J. 22: 913-924.

Aphasizhev, R., Aphasizheva, I., Nelson, R.E., and Simpson, L. 2003b. A $100-\mathrm{kD}$ complex of two RNA-binding proteins from mitochondria of Leishmania tarentolae catalyzes RNA annealing and interacts with several RNA editing components. RNA 9: 62-76.

Blom, D., Burg, J., Breek, C.K., Speijer, D., Muijsers, A.O., and Benne, R. 2001. Cloning and characterization of two guide RNAbinding proteins from mitochondria of Crithidia fasciculata: gBP27, a novel protein, and gBP29, the orthologue of Trypanosoma brucei gBP21. Nucleic Acids Res. 29: 2950-2962.

Brecht, M., Niemann, M., Schluter, E., Muller, U.F., Stuart, K., and Goringer, H.U. 2005. TbMP42, a protein component of the RNA editing complex in African trypanosomes, has endo-exoribonuclease activity. Mol. Cell 17: 621-630.

Carnes, J., Raffaello, T., Lewis Ernst, N., Steiberg, A., and Stuart, K. 2005. An essential RNase III insertion editing endonuclease in Trypanosoma brucei. Proc. Natl. Acad. Sci. 102: 16614-16619.

Cruz-Reyes, J. and Sollner-Webb, B. 1996. Trypanosome U-deletional RNA editing involves guide RNA-directed endonuclease cleavage, terminal U exonuclease, and RNA ligase activities. Proc. Natl. Acad. Sci. 93: 8901-8906.

Cruz-Reyes, J., Rusche, L.N., Piller, K.J., and Sollner-Webb, B. 1998a. T. brucei RNA editing: Adenosine nucleotides inversely affect $\mathrm{U}$-deletion and $\mathrm{U}$-insertion reactions at mRNA cleavage. Mol. Cell 1: 401-409.

Cruz-Reyes, J., Rusche, L.N., and Sollner-Webb, B. 1998b. Trypanosoma brucei $\mathrm{U}$ insertion and $\mathrm{U}$ deletion activities co-purify with an enzymatic editing complex but are differentially optimized. Nucleic Acids Res. 26: 3634-3639.

Cruz-Reyes, J., Zhelonkina, A., Rusche, L., and Sollner-Webb, B. 2001. Trypanosome RNA editing: Simple guide RNA features enhance U deletion 100-fold. Mol. Cell. Biol. 21: 884-892.

Cruz-Reyes, J., Zhelonkina, A.G., Huang, C.E., and Sollner-Webb, B. 2002. Distinct functions of two RNA ligases in active Trypanosoma brucei RNA editing complexes. Mol. Cell. Biol. 22: 4652-4660.

Ernst, N.L., Panicucci, B., Igo Jr., R.P., Panigrahi, A.K., Salavati, R., and Stuart, K. 2003. TbMP57 is a $3^{\prime}$ terminal uridylyl transferase (TUTase) of the Trypanosoma brucei editosome. Mol. Cell 11: $1525-1536$.

Grams, J., McManus, M.T., and Hajduk, S.L. 2000. Processing of polycistronic guide RNAs is associated with RNA editing complexes in Trypanosoma brucei. EMBO J. 19: 5525-5532.

Halbig, K., De Nova-Ocampo, M., and Cruz-Reyes, J. 2004. Complete cycles of bloodstream trypanosome RNA editing in vitro. RNA 10: 914-920.

Harris, M.E. and Hajduk, S.L. 1992. Kinetoplastid RNA editing: In vitro formation of cytochrome b gRNA-mRNA chimeras from synthetic substrate RNAs. Cell 68: 1091-1099.

Hema, M. and Kao, C.C. 2004. Template sequence near the initiation nucleotide can modulate brown mosaic virus RNA accumulation in plant protoplasts. J. Virol. 78: 1169-1180.

Huang, C.E., Cruz-Reyes, J., Zhelonkina, A.G., O'Hearn, S., Wirtz, E., and Sollner-Webb, B. 2001. Roles for ligases in the RNA editing complex of Trypanosoma brucei Band IV is needed for U-deletion and RNA repair. EMBO J. 20: 4694-4703.

Huang, C.E., O'Hearn, S.F., and Sollner-Webb, B. 2002. Assembly and function of the RNA editing complex in Trypanosoma brucei requires band III protein. Mol. Cell. Biol. 22: 3194-3203.

Kang, X., Falick, A.M., Nelson, R.E., Gao, G., Rogers, K., Aphasizhev, R., and Simpson, L. 2004. Disruption of the zinc finger motifs in the Leishmania tarentolae LC-4 (=TbMP63) L-complex editing protein affects the stability of the L-complex. J. Biol. Chem. 279: 3893-3899.
Kang, X., Kestrel, R., Gao, G., Falick, A.M., Zhou, S., and Simpson, L. 2005. Reconstitution of uridine-deletion pre-cleaved RNA editing with two recombinant enzymes. Proc. Natl. Acad. Sci. 102: 10171022.

Koslowsky, D.J. and Yahampath, G. 1997. Mitochondrial mRNA 3' cleavage/polyadenylation and RNA editing in Trypanosoma brucei are independent events. Mol. Biochem. Parasitol. 90: 81-94.

Law, J.A., Huang, C.E., O’Hearn, S.F., and Sollner-Webb, B. 2005. Trypanosoma brucei RNA editing, band II enables recognition specifically at each step of the U insertion cycle. Mol. Cell. Biol. 25: 2785-2794.

Leung, S.S. and Koslowsky, D.J. 2001. Interactions of mRNAs and gRNAs involved in trypanosome mitochondrial RNA editing: Structure probing of an mRNA bound to its cognate gRNA. RNA 7: 1803-1816.

Madison-Antenucci, S., Sabatini, R.S., Pollard, V.W., and Hajduk, S.L. 1998. Kinetoplastid RNA-editing-associated protein 1 (REAP-1): A novel editing complex protein with repetitive domains. EMBO J. 17: 6368-6376.

Madison-Antenucci, S., Grams, J., and Hajduk, S.L. 2002. Editing machines: The complexities of trypanosome RNA editing. Cell 108: $435-438$.

McManus, M.T., Shimamura, M., Grams, J., and Hajduk, S.L. 2001. Identification of candidate mitochondrial RNA editing ligases from Trypanosoma brucei. RNA 7: 167-175.

Milligan, J.F., Groebe, D.R., Witherell, G.W., and Uhlenbeck, O.C. 1987. Oligoribonucleotide synthesis using T7 RNA polymerase and synthetic DNA templates. Nucleic Acids Res. 15: 8783-8798.

Missel, A., Souza, A.E., Norskau, G., and Goringer, H.U. 1997. Disruption of a gene encoding a novel mitochondrial DEADbox protein in Trypanosoma brucei affects edited mRNAs. Mol. Cell. Biol. 17: 4895-4903.

Muller, U.F., Lambert, L., and Goringer, H.U. 2001. Annealing of RNA editing substrates facilitated by guide RNA-binding protein gBP21. EMBO J. 20: 1394-1404.

Oppegard, L.M. and Connell, G.J. 2002. Direct visualization of RNA editing within a Leishmania tarentolae mitochondrial extract. Int. J. Parasitol. 32: 859-866.

Oppegard, L.M., Hillestad, M., McCarthy, R.T., Pai, R.D., and Connell, G.J. 2003. Cis-acting elements stimulating kinetoplastid guide RNA-directed editing. J. Biol. Chem. 278: 51167-511175.

Pai, R.D., Oppegard, L.M., and Connell, G.J. 2003. Sequence and structural requirements for optimal guide RNA-directed insertional editing within Leishmania tarentolae. RNA 9: 469-483.

Panigrahi, A.K., Gygi, S.P., Ernst, N.L., Igo Jr., R.P., Palazzo, S.S., Schnaufer, A., Weston, D.S., Carmean, N., Salavati, R., Aebersold, R., et al. 2001a. Association of two novel proteins, TbMP52 and TbMP48, with the Trypanosoma brucei RNA editing complex. Mol. Cell. Biol. 21: 380-389.

Panigrahi, A.K., Schnaufer, A., Carmean, N., Igo Jr., R.P., Gygi, S.P., Ernst, N.L., Palazzo, S.S., Weston, D.S., Aebersold, R., Salavati, R., et al. 2001b. Four related proteins of the Trypanosoma brucei RNA editing complex. Mol. Cell. Biol. 21: 6833-6840.

Panigrahi, A.K., Schnaufer, A., Ernst, N.L., Wang, B., Carmean, N., Salavati, R., and Stuart, K. 2003. Identification of novel components of Trypanosoma brucei editosomes. RNA 9: 484-492.

Pelletier, M. and Read, L.K. 2003. RBP16 is a multifunctional gene regulatory protein involved in editing and stabilization of specific mitochondrial mRNAs in Trypanosoma brucei. RNA 9: 457-468.

Reichert, V.L., Le Hir, H., Jurica, M.S., and Moore, M.J. 2002. 5'exon interactions within the human spliceosome establish a framework for exon junction complex structure and assembly. Genes \& Dev. 16: $2778-2791$.

Rusche, L.N., Cruz-Reyes, J., Piller, K.J., and Sollner-Webb, B. 1997. Purification of a functional enzymatic editing complex from Trypanosoma brucei mitochondria. EMBO J. 16: 4069-4081.

Rusche, L.N., Huang, C.E., Piller, K.J., Hemann, M., Wirtz, E., and Sollner-Webb, B. 2001. The two RNA ligases of the Trypanosoma brucei RNA editing complex: Cloning the essential band IV gene and identifying the band V gene. Mol. Cell. Biol. 21: 979-989. 
Sabatini, R. and Hajduk, S.L. 1995. RNA ligase and its involvement in guide RNA/mRNA chimera formation. Evidence for a cleavage-ligation mechanism of Trypanosoma brucei mRNA editing. J. Biol. Chem. 270: 7233-7240.

Schnaufer, A., Panigrahi, A.K., Panicucci, B., Igo Jr., R.P., Wirtz, E., Salavati, R., and Stuart, K. 2001. An RNA ligase essential for RNA editing and survival of the bloodstream form of Trypanosoma brucei. Science 291: 2159-2162.

Schnaufer, A., Ernst, N.L., Palazzo, S.S., O’Rear, J., Salavati, R., and Stuart, K. 2003. Separate insertion and deletion subcomplexes of the Trypanosoma brucei RNA editing complex. Mol. Cell 12: 307319.

Seiwert, S.D., Heidmann, S., and Stuart, K. 1996. Direct visualization of uridylate deletion in vitro suggests a mechanism for kinetoplastid RNA editing. Cell 84: 831-841.

Simpson, L., Aphasizhev, R., Gao, G., and Kang, X. 2004. Mitochondrial proteins and complexes in Leishmania and Trypanosoma involved in U-insertion/deletion RNA editing. RNA 10: 159-170.

Sollner-Webb, B., Rusche, L.N., and Cruz-Reyes, J. 2001. Ribonuclease activities of trypanosome RNA editing complex directed to cleave specifically at a chosen site. Methods Enzymol. 341: 154-174.

Stuart, K.D., Schnaufer, A., Ernst, N.L., and Panigrahi, A.K. 2005. Complex management: RNA editing in trypanosomes. Trends Biochem. Sci. 30: 97-105.

Suck, D. 1997. Common fold, common function, common origin? Nat. Struct. Biol. 4: 161-165.

Trotter, J.R., Ernst, N.L., Carnes, J., Panicucci, B., and Stuart, K. 2005. A deletion site editing endonuclease in Trypanosoma brucei. Mol. Cell 20: 403-412.

Vanhamme, L., Perez-Morga, D., Marchal, C., Speijer, D., Lambert, L., Geuskens, M., Alexandre, S., Ismaili, N., Goringer, U., and Benne, R.1998. Trypanosoma brucei TBRGG1, a mitochondrial oligo(U)-binding protein that co-localizes with an in vitro RNA editing activity. J. Biol. Chem. 273: 21825-21833.

Vondruskova, E., van den Burg, J., Zikova, A., Ernst, N.L., Stuart, K., Benne, R., and Lukes, J. 2005. RNA interference analyses suggest a transcript-specific regulatory role for mitochondrial RNAbinding proteins MRP1 and MRP2 in RNA editing and other RNA processing in Trypanosoma brucei. J. Biol. Chem. 280: 2429-2438. 

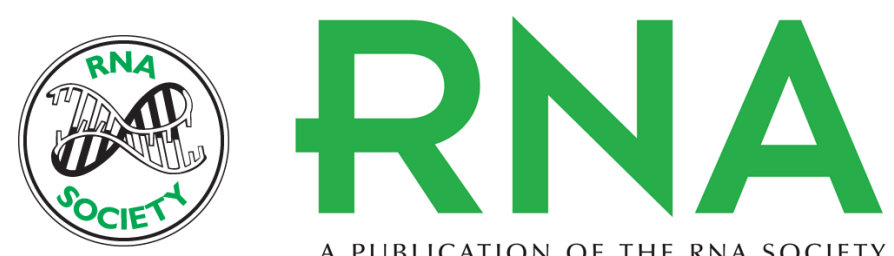

A PUBLICATION OF THE RNA SOCIETY

\section{RNA editing complex interactions with a site for full-round $U$ deletion in Trypanosoma brucei}

Anastasia Sacharidou, Catherine Cifuentes-Rojas, Kari Halbig, et al.

RNA 2006 12: 1219-1228

References This article cites 50 articles, 33 of which can be accessed free at: http://rnajournal.cshlp.org/content/12/7/1219.full.html\#ref-list-1

Open Access Freely available online through the RNA Open Access option.

License Freely available online through the open access option.

Email Alerting Receive free email alerts when new articles cite this article - sign up in the box at the Service top right corner of the article or click here. 